\title{
Impact of historic histopathologic sample review on the risk of recurrence in patients with differentiated thyroid cancer
}

Fabián Pitoia', Fernando Jerkovich', Carolina Urciuoli', Florencia Falcón², Andrea Páes de Lima²

\begin{abstract}
Objective: To compare the historic risk of recurrence (RR) and response to therapy to risk stratification estimated with historical pathology reports (HPRs) and contemporary re-review of the pathological slides in patients with differentiated thyroid cancer (DTC). Subjects and methods: Out of 210 DTC patients with low and intermediate RR who underwent total thyroidectomy and remnant ablation in our hospital, 63 available historic pathologic samples (HPS) were reviewed. The RR and the response to therapy were evaluated considering historical histological features (histological type, tumor size, capsular invasion, number of lymph node metastases) and then, reassessed after observing additional histological features (vascular invasion, extrathyroidal extension, size of lymph node metastases, presence of extranodal extension, and/or status of the resection margins). Results: A change in the RR category was observed in 16 of 63 cases (25.4\%). Out of 46 patients initially classified as low $R R$, 2 patients were reclassified as intermediate RR, 4 as high $R R$, and 1 as noninvasive follicular thyroid neoplasm with papillary-like nuclear features (NIFTP). Out of 17 patients initially classified as intermediate RR, 3 were reassigned to the low RR group, 5 as high $R R$, and 1 as NIFTP.The percentages of structural incomplete response at final follow-up changed from 2.2 to $0 \%(p=1)$ in patients with low RR and from 6.3 to $20 \%(p=0.53)$ in patients with intermediate RR. Conclusion: A detailed report of specific features in the HPR of patients with DTC might give a more accurate RR classification and a better estimation of the response to treatment. Arch Endocrinol Metab. 2018;62(2):157-63
\end{abstract}

Division of Endocrinology, University of Buenos Aires Buenos Aires, Argentina ${ }^{2}$ Department of Pathology, University of Buenos Aires Buenos Aires, Argentina

\section{Correspondence to: Fabián Pitoia \\ División Endocrinología Hospital de Clínicas, University of Buenos Aires Córdoba 2351, $5^{\text {th }}$ Floor, Buenos Aires 1001, Argentina fpitoia@intramed.net}

Received on Aug/24/2017 Accepted on Nov/8/2017

DOI: $10.20945 / 2359-3997000000020$

\section{INTRODUCTION}

$\mathrm{T}$ he pathological examination of thyroid samples is important for establishing the diagnosis of differentiated thyroid carcinoma (DTC), and it also provides useful information for the risk stratification assessment (1). On the other side, the TNM AJCC/ UICC staging system is recommended for predicting disease mortality ( 1 ).

The 2009 version of the American Thyroid Association (ATA) thyroid cancer guidelines proposed a three-tiered risk stratification system in which specific clinical-pathologic features are used to estimate the risk of structural incomplete response (SIR) and the probability of an excellent response to treatment (2). The variables taken into account for risk stratification in the 2009 version of the ATA guidelines were: evidence of microscopic or gross extrathyroidal extension, presence of aggressive histology, completeness of tumor resection, existence of metastatic lymph node/s and/or distant metastasis, and observation of RAI-avid disease outside the thyroid bed (2).

In this first ATA risk of recurrence (RR) classification system, many important features were not taken into account. For example, any patient with lymph node metastasis was deemed to have an intermediate RR. In fact, the risk of structural disease can vary from $4 \%$ if having fewer than five metastatic lymph nodes, or $5 \%$ if all involved lymph nodes are less than $0.2 \mathrm{~cm}$, to $19 \%$ if more than five lymph nodes are involved or $27 \%-32 \%$ if any metastasis in the lymph node is larger than $3 \mathrm{~cm}$ $(3,4)$. Additionally, follicular thyroid carcinomas (FTCs) with vascular invasion, initially classified by the 2009 ATA system as intermediate $\mathrm{RR}$, have an excellent prognosis when minor vascular invasion is present but may have a high RR (30\%-55\%) when more than four foci are demonstrated in the pathological examination $(5,6)$. 
Although the 2009 risk stratification system has proven to be a valuable tool for initial risk stratification in DTC (7-10), modifications were required to better assess the RR of DTC patients. The recently published 2015 version of the ATA guidelines included specific prognostic variables: the extent of vascular invasion, the extent of lymph node involvement (number and size of metastatic lymph nodes and presence of extranodal extension), and mutational status (BRAF and/or TERT when available) (1). As a result, the low risk category was expanded to include patients with small-volume lymph node metastases (clinical N0 or $<5$ pathologic $\mathrm{Nl}$ micrometastases, $<0.2 \mathrm{~cm}$ in the largest dimension), the intermediate RR group now considers only a subset of patients with lymph node metastases (clinical $\mathrm{Nl}$ or $>5$ pathologic $\mathrm{Nl}$ with all involved lymph nodes $<3$ $\mathrm{cm}$ in the largest dimension), and a second group, with intermediate to high risk of recurrence, now includes those cases with large-volume lymph node involvement (any metastatic lymph node $>3 \mathrm{~cm}$ in the largest dimension, $>3$ lymph node metastasis with extranodal extension) and FTC with extensive vascular invasion ( $>4$ foci of vascular invasion or extracapsular vascular invasion). The 2015 guidelines defined this new version of the RR as the "modified risk stratification system from ATA 2009 guidelines" (MRSS ATA 2009) and were validated by our and other groups around the world in ablated and non-ablated patients (11-14).

The vascular invasion $(\geq$ or $<4$ foci), the magnitude of extrathyroidal extension, the size of the largest metastatic focus in lymph nodes, the presence of extranodal extension, and the status of the resection margins were not uniformly informed in the historic pathologic reports that were used to assess the RR of DTC patients in our hospital and most centers in Latin America, with the exception of the number of lymph node metastases.

However, the additional benefit of adding the previously mentioned specific features for reclassifying patients has not yet been established. Therefore, our objective was to compare the historic RR and the responses to treatment to risk stratification systems estimated with historical pathology reports and contemporary re-review of the slides by two expert pathologists.

\section{SUBJECTS AND METHODS}

\section{Inclusion criteria}

The file records from our database of 610 patients with DTC were reviewed for the period January 2001 to
March 2016. The inclusion criteria were i) to be 18 years old or older, ii) to have had a follow-up for at least 3 years, iii) to have received a total thyroidectomy with or without lymph node dissection and remnant ablation with radioiodine after thyroid hormone withdrawal (THW) or recombinant human thyrotropin (rhTSH). Lymph node dissection was performed when clinical pathological lymph nodes were diagnosed previous to surgery, when the surgeons had a suspicion of lymph node metastasis, or when the size of the thyroid tumor was greater than $4 \mathrm{~cm}$ in diameter.

Out of 268 patients who met these inclusion criteria, 210 patients with low and intermediate $R R$ were considered for analysis. The RR was assessed as described in Table 1. Because the historic pathological reports did not systematically detail the size of the lymph node metastasis, the extent of extranodal extension, the presence of vascular invasion, or the microscopic invasion of surgical margins, these variables were not taken into account for the initial RR stratification of these patients. The number of metastatic lymph nodes ( $>5$, intermediate $\mathrm{RR}$, and $<5$ low $\mathrm{RR}$ ), as it was available in the pathological report, was considered for the initial RR classification. Also, as it was validated in a previous analysis from our cohort of DTC patients in our institution, intrathyroidal tumors with minor extrathyroidal extension (T3) were considered in the low RR group (11). One hundred fifteen patients were excluded because they had been operated on in other institutions and the pathological slides were not available in our hospital. Also, 32 patients were excluded due to insufficient histopathological material.

Sixty-three low and intermediate RR patients were included in the final analysis. The historic histopathologic samples (HPS) of these 63 patients were reviewed by two senior pathologists from our hospital (P.L.A. and F.F.). The analysis consisted of a first diagnosis provided by F.F. and a re-review and agreement with P.L.A., to give a final homogeneous result.

\section{Pathological analysis}

The RR was reassessed considering the following variables: histologic variant, number of foci of vascular invasion, volume of lymph node metastases (number of metastatic lymph nodes and size), presence of extranodal extension, and status of the resection margins. The RR classification according to these new histological features can be seen in Table 1 . 
Table 1. Risk of recurrence classification considering specific histological features

\begin{tabular}{ll}
\hline Low RR & Classical and follicular variant PTC \\
& Minor extrathyroidal extension \\
& $<4$ foci of vascular invasion \\
& $<5 \mathrm{LN}$ metastasis $0.2-3 \mathrm{~cm}$ \\
& All LN metastasis $<0.2 \mathrm{~cm}$ \\
& No evidence of extranodal extension \\
& Aggressive histology (tall cell, columnar cell, \\
Intermediate RR & Hürthle cell carcinomas, oncocytic PTC) \\
& $>5$ LN metastasis $0.2-3 \mathrm{~cm}$ \\
& Extranodal extension in $<3$ LN metastasis \\
& $\geq 4$ foci of vascular invasion \\
Intermediate-high RR & Extranodal extension in $>3$ LN metastasis \\
& Any LN $>3 \mathrm{~cm}$ \\
& Surgical margins involved \\
\hline
\end{tabular}

RR: risk of recurrence; PTC: papillary thyroid cancer; LN: lymph node.

Patients having an encapsulated follicular variant of papillary thyroid carcinoma (FVPTC) were reclassified as noninvasive follicular thyroid neoplasm with papillarylike nuclear features (NIFTP), currently considered as a benign neoplasm according to the criteria proposed by Nikoforov and cols. (15).

\section{Ablation protocol}

Patients received radioiodine ablation after stimulation with rhTSH $(n=31)$ or endogenous TSH $(n=32)$, using activities of $3.70 \mathrm{GBq}(100 \mathrm{mCi} 131-\mathrm{I})$ for low risk (ATA $2009 \mathrm{RR}$, Table $\mathrm{l}$ ) and 5.55 GBq (150 $\mathrm{mCi}$ 131-I) for intermediate risk (ATA $2009 \mathrm{RR}$, Table 1). Endogenous TSH stimulation was obtained after thyroid hormone withdrawal for at least 3 weeks. Radioiodine was administered following that interval, in all cases with TSH levels above $50 \mathrm{mUI} / \mathrm{L}$.

\section{Clinical endpoints}

The initial response to therapy, defined as the best response to initial therapy assessed at the $12 \pm 5$ months visit, and the clinical status at final follow-up were evaluated according to the response-to-therapy classification proposed by the ATA 2015 guidelines (2). The initial and final responses were compared to the risk stratification before and after reviewing the pathological samples.

\section{Statistical analysis}

Quantitative variables were expressed as the mean \pm standard error mean, median, and range, while qualitative variables were shown as percentages. To evaluate significant differences in the percentages of the responses-to-therapy before and after the pathologic review, we analyzed two-way contingency tables by the Fisher exact test or $2 \times 2$ contingency table by the chisquare test when appropriate. Statistical analysis was developed using the SPSS statistical software (SPSS version 20.0, Inc., Chicago, IL, USA). We considered $p<0.05$ to be statistically significant for all the analyses.

\section{RESULTS}

The demographics, clinical features, and MRSS ATA 2009 for each of the 63 patients included in this study are seen in Table 2.

Table 2. Baseline characteristics of 63 patients with DTC included in the study

\begin{tabular}{lc}
\hline Gender & \\
Female/Male & $58(92 \%) / 5(8 \%)$ \\
Age (years) & \\
Mean \pm SEM & $48.3 \pm 16.1$ \\
Median (range) & $47(21.2-76)$ \\
Histology & \\
PTC classic variant & $47(74.6 \%)$ \\
PTC follicular variant & $13(20.6 \%)$ \\
PTC oncocytic variant & $2(3.1 \%)$ \\
PTC tall cell variant & $1(1.7 \%)$ \\
Cervical lymphadenectomy & \\
No & $18(28.5 \%)$ \\
Central & $40(63.4 \%)$ \\
Central and lateral & $5(9.1 \%)$ \\
Bilaterality, multifocality, and capsular invasion & \\
Bilateral & $18(28.5 \%)$ \\
Multifocal & $13(20.6 \%)$ \\
Capsular invasion & $16(25.3 \%)$ \\
TNM stage & \\
I & \\
II & \\
III & $45(71.4 \%)$ \\
MRSS ATA 2009 before HSR & $1(1.7 \%)$ \\
Low & $17(26.9 \%)$ \\
Intermediate & \\
131I Cumulative Dose (mCi) & $46(73 \%)$ \\
Mean \pm SEM & $17(27 \%)$ \\
Median (range) & \\
\hline
\end{tabular}

MRSS ATA 2009: modified risk stratification system from ATA 2009 guidelines; SEM: standard error mean.

The percentages of new specific histological features assessed after the pathological review are detailed in Table 3. 
Table 3. Characteristics of initial pathological reports compared with those obtained after histopathological sample review

\begin{tabular}{lcc}
\hline Variable & $\begin{array}{c}\text { Informed in } \\
\text { initial report }\end{array}$ & $\begin{array}{c}\text { Informed } \\
\text { after HSR }\end{array}$ \\
\hline $\begin{array}{l}\text { Number of foci of vascular invasion }(>4) \\
\text { Surgical margins involved }\end{array}$ & Not informed & $0 / 63(0 \%)$ \\
Metastatic lymph node size & Not informed & $7 / 63(11.1 \%)$ \\
$<0.2 \mathrm{~cm}$ & & \\
$0.2-1 \mathrm{~cm}$ & & $5 / 19(31.6 \%)$ \\
$>1 \mathrm{~cm}<3 \mathrm{~cm}$ & & $5 / 19(26.3 \%)$ \\
$>3 \mathrm{~cm}$ & Not informed & $3 / 19(19.3 \%)$ \\
Extranodal extension & & $1 / 19(5.8 \%)$ \\
$>3$ lymph nodes & $18(28.5 \%)$ & $18(28.5 \%)$ \\
Multifocality & $13(20.6 \%)$ & $13(20.6 \%)$ \\
Bilaterality & Not informed & $0 / 63(0 \%)$ \\
Necrosis & $16 / 63(25.6 \%)$ & \\
Extrathyroidal extension & Not informed & $24 / 63(38 \%)$ \\
$\quad$ Minimal & Not informed & $7 / 63(11.1 \%)$ \\
\hline Gross $>1 \mathrm{~cm})$ & & \\
\hline
\end{tabular}

HSR: histopathological sample review.

After the review of the pathologic slides, a change in the RR category was made in $25.4 \%(16 / 63)$ of the cases. Out of 46 patients initially classified as low RR, 2 patients were reclassified as intermediate $R R$ because of extranodal extension in fewer than 3 lymph nodes, 4 patients were reassigned to the intermediate-high RR group because of surgical margins involved, and 1 patient finally was diagnosed as harboring an NIFTP (Table 4).

Out of 17 patients initially classified as intermediate $\mathrm{RR}, 3$ were reassigned to the low RR group ( 1 because of a change in the histology variant and 2 due to the presence of micrometastasis in all involved lymph nodes) whereas 5 patients were reclassified to the intermediate-high category (4 because of surgical margins involved and 1 because more than 3 metastatic lymph nodes with extranodal extension were present). Three patients with positive surgical margins also had at least 1 lymph node metastasis $>3 \mathrm{~cm}$ in the largest dimension. One patient who initially was assigned to the intermediate RR group was diagnosed as NIFTP (Table 4).

Finally, the percentages of initial response to therapy and the final clinical outcomes were compared between the initial RR assessed before and after the pathology slide review. The percentage of SIR at initial evaluation changed from 6.7 to $2.4 \%(p=0.6254)$ in the low risk group and from 12.5 to $20 \%(p=0.6171)$ in the intermediate risk group (Table 5 ). The frequency of SIR at final follow-up changed from 2.2 to $0 \%(p=1)$ in patients with low RR and from 6.3 to $20 \%$ ( $p=0.5304$ ) in patients with intermediate RR (Table 6).

\section{DISCUSSION}

A detailed pathology review of the historic histological samples of 63 DTC patients initially classified as low or intermediate $R R$ by a validated risk stratification system (7-11) demonstrated that, considering specific features (extent of extranodal extension, extent of vascular invasion, and surgical margin evaluation, among others), the change in percentage of SIR at initial and final evaluation did not significantly differ from the statistical point of view. However, adding these

Table 4. Reasons for reclassification of patients after the historic pathology slide review

\begin{tabular}{|c|c|c|}
\hline MRSS ATA 2009 & MRSS ATA 2009 after reviewing the HPS & Reason for changing the RR \\
\hline \multirow[t]{4}{*}{ Low $\operatorname{RR}(n=46)$} & Low $\operatorname{RR}(n=39)$ & \\
\hline & Intermediate RR $(n=2)$ & $\begin{array}{l}n=1 \text {, PTC oncocytic variant (previously classic variant PTC) } \\
n=1 \text {, extranodal extension in }<3 \text { LN metastasis }\end{array}$ \\
\hline & Intermediate-high RR $(n=4)$ & $\begin{array}{l}n=3, \text { microscopic invasion of surgical margins } \\
n=1,40 \% \text { of tumor dedifferentiation }\end{array}$ \\
\hline & $\operatorname{NIFTP}(n=1)$ & \\
\hline \multirow[t]{4}{*}{ Intermediate RR $(n=17)$} & Low $\operatorname{RR}(n=3)$ & $\begin{array}{l}n=1, \text { classic PTC (previously PTC tall cell variant) } \\
n=2, \text { all LN metastasis }<0.2 \mathrm{~cm}\end{array}$ \\
\hline & Intermediate RR $(n=8)$ & \\
\hline & Intermediate-high RR ( $n=5)$ & $\begin{array}{l}n=4 \text {, microscopic invasion of surgical margins }(n=3 \text { of them with } \mathrm{LN} \text { metastasis }>3 \mathrm{~cm} \text { ) } \\
n=1 \text {, extranodal extension in }>3 \mathrm{LN} \text { metastases }\end{array}$ \\
\hline & $\operatorname{NIFTP}(n=1)$ & \\
\hline
\end{tabular}

MRSS ATA 2009: modified risk stratification system from ATA 2009 guidelines; RR: risk of recurrence; NIFTP: noninvasive follicular thyroid neoplasm with papillary-like nuclear features; HPS: historic pathology slide; PTC: papillary thyroid carcinoma; LN: Iymph node. 
Table 5. Comparison of the initial response to therapy to risk of recurrence assessed before and after HPS review

\begin{tabular}{|c|c|c|c|c|}
\hline & \multicolumn{4}{|c|}{ Initial response to therapy $(n=61)^{\mathrm{a}}$} \\
\hline & Excellent & Indeterminate & Biochemical incomplete & Structural incomplete \\
\hline \multicolumn{5}{|l|}{ MRSS ATA 2009} \\
\hline Low $\operatorname{RR}(n=45)$ & $27(60 \%)$ & $14(31.1 \%)$ & $1(2.2 \%)$ & $3(6.7 \%)$ \\
\hline Intermediate $\mathrm{RR}(n=16)$ & $6(37.5 \%)$ & $4(25 \%)$ & $4(25 \%)$ & $2(12.5 \%)$ \\
\hline \multicolumn{5}{|l|}{ MRSS ATA 2009 after HPS reviewa } \\
\hline Low $\operatorname{RR}(n=42)$ & $30(71.4 \%)^{b}$ & $10(23.8 \%)$ & $1(2.4 \%)$ & $1(2.4 \%)^{b}$ \\
\hline Intermediate $\operatorname{RR}(n=10)$ & $4(40 \%)^{c}$ & $0(0 \%)$ & $4(40 \%)$ & $2(20 \%)^{c}$ \\
\hline Intermediate-high RR $(n=9)$ & $3(33.3 \%)$ & $1(11.1 \%)$ & $3(33.3 \%)$ & $2(22.3 \%)$ \\
\hline
\end{tabular}

MRSS ATA 2009: modified risk stratification system from ATA 2009 guidelines; RR: risk of recurrence; NIFPT: noninvasive follicular thyroid neoplasm with papillary-like nuclear features; HPS: historic pathology slide.

a $n=2$ patients reclassified as NIFTP were excluded from the analysis. They both had an initial excellent response.

${ }^{b}$ Comparison between risk of recurrence classification before and after historic pathology slide review in low risk patients: excellent response (60\% vs. $\left.71.4 \%, p=0.3668\right)$, structural incomplete response $(6.7 \%$ vs. $2.4 \%, p=0.6171)$.

' Comparison between risk of recurrence classification before and after historic pathology slide review in intermediate risk patients: excellent response ( $37.5 \%$ vs. $40 \%, p=1)$, structural incomplete response $(12.5 \%$ vs. $20 \%, p=0.6254)$.

Table 6. Comparison of the final response to therapy between risk of recurrence assessed before and after HPS review

\begin{tabular}{|c|c|c|c|c|}
\hline & \multicolumn{4}{|c|}{ Final response to therapya $(n=61)$} \\
\hline & Excellent & Indeterminate & Biochemical incomplete & Structural incomplete \\
\hline \multicolumn{5}{|l|}{ MRSS ATA 2009} \\
\hline Low $\mathrm{RR}(n=45)$ & $31(68.9 \%)$ & $12(26.7 \%)$ & $1(2.2 \%)$ & $1(2.2 \%)$ \\
\hline Intermediate RR $(n=16)$ & $7(43.7 \%)$ & $6(37.5 \%)$ & $2(12.5 \%)$ & $1(6.3 \%)$ \\
\hline \multicolumn{5}{|l|}{ MRSS ATA 2009 after HPS reviewa } \\
\hline Low $\operatorname{RR}(n=42)$ & $31(73.8 \%)^{b}$ & $10(23.8 \%)$ & $1(2.4 \%)$ & $0(0 \%)^{b}$ \\
\hline Intermediate $\mathrm{RR}(n=10)$ & $3(30 \%)^{c}$ & $3(30 \%)$ & $2(20 \%)$ & $2(20 \%)^{c}$ \\
\hline Intermediate-high RR $(n=9)$ & $3(33.33 \%)$ & $3(33.33 \%)$ & $3(33.33 \%)$ & $0(0 \%)$ \\
\hline
\end{tabular}

MRSS ATA 2009: modified risk stratification system from ATA 2009 guidelines; RR: risk of recurrence; NIFTP: noninvasive follicular thyroid neoplasm with papillary-like nuclear features; HPS: historic pathology slide.

Follow-up of the entire cohort (months): mean $42.6 \pm 3.3$, median 39.3 (range 36.1-67.9).

a 2 patients reclassified as NIFPT were excluded from the analysis. They had excellent response at final outcome (36 and 38 months of follow-up).

${ }^{b}$ Comparison between risk of recurrence classification before and after historic pathology slide review in low risk patients: excellent response (68.9\% vs. $\left.73.8 \%, p=0.6431\right)$, structural incomplete response $(2.2 \%$ vs. $0 \%, p=1)$.

${ }^{\circ}$ Comparison between risk of recurrence classification before and after historic pathology slide review in intermediate risk patients: excellent response $(43.5 \%$ vs. $30 \%, p=0.6913)$, structural incomplete response (6.3\% vs. $20 \%, p=0.5304)$.

new variables to the RR classification might confer a more accurate estimation of the probability of having an SIR at final follow-up. This frequency was $6.3 \%$ in the intermediate RR group previous to the pathologic review and 20\% after adding specific histological data, which is more similar to what it was reported by larger cohorts of DTC patients $(21 \%-34 \%)$ (7-11). Also, the excellent response at final outcome was achieved in $68.9 \%$ of low RR patients, but when patients were reclassified after the pathologic review, this percentage rose to $73.8 \%$ (without statistical difference).

Two patients from this cohort were diagnosed with the encapsulated variant of follicular thyroid carcinoma, which is currently known as NIFTP $(15,16)$. These tumors are characterized by a follicular growth pattern without papillae formation, nuclear features of papillary thyroid carcinoma, encapsulation or clear demarcation, and no vascular or capsular invasion (15). They are associated with an indolent behavior and a very low risk of adverse outcome (15). The encapsulated forms of the follicular variant of PTC also have a distinct molecular profile, harboring predominantly RAS mutations in contrast with the more frequent $B R A F$ mutations in the infiltrative forms $(17,18)$. Currently, NIFTP is assumed to represent nearly two-thirds of the follicular variant PTCs $(15,19,20)$. Although the ATA 2015 task force has recently proposed that this neoplasm with very low potential for recurrence should be reclassified as a non-cancer (16), and the 2 patients with NIFTP from our cohort had an excellent response to therapy 
(both treated with total thyroidectomy and radioiodine ablation), isolated cases of distant metastasis have been reported (21-23).

It is noteworthy that 4 patients initially classified as having a low RR were reassigned to the intermediatehigh RR group. Although it is difficult to draw a conclusion with this small cohort of patients $(n=$ 9 ), this subgroup of patients showed $22 \%$ of SIR at initial evaluation, similar to what is expected in the intermediate RR group of patients (7-11). Indeed, all of them achieved an excellent response at final follow-up. Therefore, it seems that the presence of surgical margins involved in the microscopic analysis, the presence of extranodal extension in more than 3 metastatic lymph nodes, and $<50 \%$ of tumor dedifferentiation would probably have less impact on the percentage of SIR than the macroscopic invasion of tumor into the perithyroidal soft tissues or the presence of distant metastasis (1,7-10). It seems that, in the absence of extrathyroidal extension in small primary tumors, the positive margin status does not appear to be a poor prognostic indicator for local recurrence and has an excellent local recurrence-free survival, comparable to those with negative margins, at least in patients who received radioiodine remnant ablation, as it happened in our cohort (24).

The presence of vascular invasion is recognized as a variable that has a negative impact on the RR (2527 ), and it should be informed in the pathological report. It is diagnosed as a direct tumor extension into the blood vessel lumen typically attached to the wall and covered by a fibrin thrombus (27). Minor vascular invasion (small number of foci confined to intracapsular vessels) appears to have a low risk of SIR (less than $5 \%$ ) (28-30). On the contrary, more than 4 foci of vascular invasion are associated with a poor prognosis, mainly in follicular carcinomas (30-32). This situation seems to be uncommon in papillary carcinomas, even more in micro PTC, with a prevalence of less than $4 \%$ $(25,26,28,33-35)$. In fact, none of the 63 patients from our cohort had vascular invasion in more than 4 foci in the pathological review.

In our cohort, we considered the oncocytic variant of PTC as an aggressive histology such as columnar cell and tall cell subtypes. The oncocytic change in PTC patients was reported to have a higher recurrence rate than PTC patients without oncocytic change $(30.8 \%$ vs. $11.7 \%)(36)$.
One limitation of this study is the low number of patients in each RR group, which could have limited the statistical analysis. Given $2.2 \%$ and $0 \%$ SIR in the low risk groups and 6.3 and $20 \%$ SIR in the intermediate risk groups, a type I error of 5\% (two-sided), and an interval confidence of $95 \%$, a total of 966 low risk patients and 218 intermediate risk patients would have to be included to achieve a statistical power of at least $80 \%$. It is necessary to validate these results in larger cohorts of patients. Another limitation is that every single patient in our cohort, even those classified as low risk, was treated with total thyroidectomy and RAI, a common practice in the past in most countries of Latin America (37). Currently, this practice of care may not apply to many institutions where low risk patients do not receive remnant ablation, including in our hospital (14). Therefore, further analysis is necessary to validate our results in low risk patients who do not receive RAI.

In conclusion, a detailed report of specific features in the HPR of patients with DTC might give a more accurate RR classification and a better estimation of the response to treatment. If these data are not available, the RR can also be estimated, but the expected SIR might be slightly different, although not statistically significant, as we could see in our analysis.

Disclosure: no potential conflict of interest relevant to this article was reported.

\section{REFERENCES}

1. Haugen BR, Alexander EK, Bible KC, Doherty GM, Mandel SJ, NikiforovYE, et al. 2015 American Thyroid Association Management Guidelines for Adult Patients with Thyroid Nodules and Differentiated Thyroid Cancer: The American Thyroid Association Guidelines Task Force on Thyroid Nodules and Differentiated Thyroid Cancer. Thyroid. 2016;26(1):1-133.

2. American Thyroid Association (ATA) Guidelines Taskforce on Thyroid Nodules and Differentiated Thyroid Cancer, Cooper DS, Doherty GM, Haugen BR, Kloos RT, Lee SL, Mandel SJ, et al. Revised American Thyroid Association management guidelines for patients with thyroid nodules and differentiated thyroid cancer. Thyroid. 2009;19(11):1167-214.

3. Randolph GW, Duh QY, Heller KS, LiVolsi VA, Mandel SJ, Steward $\mathrm{DL}$, et al. The prognostic significance of nodal metastases from papillary thyroid carcinoma can be stratified based on the size and number of metastatic lymph nodes, as well as the presence of extranodal extension. Thyroid. 2012;22(11):1144-52.

4. Lee J, Song Y, Soh EY. Prognostic significance of the number of metastatic lymph nodes to stratify the risk of recurrence. World J Surg. 2014;38(4):858-62.

5. Collini P, Sampietro G, Pilotti S. Extensive vascular invasion is a marker of risk of relapse in encapsulated non-Hürthle cell follicular carcinoma of the thyroid gland: a clinicopathological study 
of 18 consecutive cases from a single institution with a 11-year median follow-up. Histopathology. 2004;44(1):35-9.

6. Huang CC, Hsueh C, Liu FH, Chao TC, Lin JD. Diagnostic and therapeutic strategies for minimally and widely invasive follicular thyroid carcinomas. Surg Oncol. 2011;20(1):1-6.

7. Tuttle RM, Tala H, Shah J, Leboeuf R, Ghossein R, Gonen M, et al. Estimating risk of recurrence in differentiated thyroid cancer after total thyroidectomy and radioactive iodine remnant ablation: using response to therapy variables to modify the initial risk estimates predicted by the new American Thyroid Association staging system. Thyroid. 2010;20(12):1341-9.

8. Vaisman F, Momesso D, Bulzico DA, Pessoa CH, Dias F, Corbo R, et al. Spontaneous remission in thyroid cancer patients after biochemical incomplete response to initial therapy. Clin Endocrinol (Oxf). 2012;77(1):132-8.

9. Castagna MG, Maino F, Cipri C, Belardini V, Theodoropoulou A, Cevenini G, et al. Delayed risk stratification, to include the response to initial treatment (surgery and radioiodine ablation), has better outcome predictivity in differentiated thyroid cancer patients. Eur J Endocrinol. 2011;165(3):441-6.

10. Pitoia F, Bueno F, Urciuoli C, Abelleira E, Cross G, Tuttle RM. Outcomes of patients with differentiated thyroid cancer risk-stratified according to the American thyroid association and Latin American thyroid society risk of recurrence classification systems. Thyroid. 2013;23(11):1401-7.

11. Pitoia F, Jerkovich F, Urciuoli C, Schmidt A, Abelleira E, Bueno F, et al. Implementing the Modified 2009 American Thyroid Association Risk Stratification System in Thyroid Cancer Patients with Low and Intermediate Risk of Recurrence. Thyroid. 2015;25(11):1235-42.

12. Momesso DP, Vaisman F, Yang SP, Bulzico DA, Corbo R, Vaisman $M$, et al. Dynamic Risk Stratification in Patients with Differentiated Thyroid Cancer Treated Without Radioactive lodine. J Clin Endocrinol Metab. 2016;101(7):2692-700.

13. Park S, Kim WG, Song E, Oh HS1, Kim M, Kwon H, et al. Dynamic Risk Stratification for Predicting Recurrence in Patients with Differentiated Thyroid Cancer Treated Without Radioactive lodine Remnant Ablation Therapy. Thyroid. 2017;27(4):524-530.

14. Abelleira E, Bueno F, Smulever A, Pitoia F. Riesgo dinámico en pacientes con cáncer diferenciado de tiroides no ablacionados. Rev Argent Endocrinol Metab. 2017;54(2):69-75.

15. Nikiforov YE, Seethala RR, Tallini G, Baloch ZW, Basolo F, Thompson LD, et al. Nomenclature Revision for Encapsulated Follicular Variant of Papillary Thyroid Carcinoma: A Paradigm Shift to Reduce Overtreatment of Indolent Tumors. JAMA Oncol. 20161;2(8):1023-9.

16. Haugen BR, Sawka AM, Alexander EK, Bible KC, Caturegli P, Doherty GM, et al. American Thyroid Association Guidelines on the Management of Thyroid Nodules and Differentiated Thyroid Cancer Task Force Review and Recommendation on the Proposed Renaming of Encapsulated Follicular Variant Papillary Thyroid Carcinoma Without Invasion to Noninvasive Follicular Thyroid Neoplasm With Papillary-Like Nuclear Features. Thyroid. 2017;27(4):481-3.

17. Rivera M, Ricarte-Filho J, Knauf J, Shaha A, Tuttle M, Fagin JA, et al. Molecular genotyping of papillary thyroid carcinoma follicular variant according to its histological subtypes (encapsulated vs infiltrative) reveals distinct BRAF and RAS mutation patterns. Mod Pathol. 2010;23(9):1191-200.

18. Howitt BE, Jia Y, Sholl LM, Barletta JA. Molecular alterations in partially-encapsulated or well-circumscribed follicular variant of papillary thyroid carcinoma. Thyroid. 2013;23(10):1256-62.

19. Liu J, Singh B, Tallini G, Carlson DL, Katabi N, Shaha A, et al. Follicular variant of papillary thyroid carcinoma: a clinicopathologic study of a problematic entity. Cancer. 2006;107(6):1255-64.
20. Piana S, Frasoldati A, Di Felice E, Gardini G, Tallini G, Rosai J. Encapsulated well-differentiated follicular-patterned thyroid carcinomas do not play a significant role in the fatality rates from thyroid carcinoma. Am J Surg Pathol. 2010;34(6):868-72.

21. Baloch ZW, LiVolsi VA. Encapsulated follicular variant of papillary thyroid carcinoma with bone metastases. Mod Pathol. 2000;13(8):861-5.

22. Hunt JL, Dacic S, Barnes EL, Bures JC. Encapsulated follicular variant of papillary thyroid carcinoma. Am J Clin Pathol. 2002;118(4):602-3.

23. Baloch ZW, Shafique K, Flannagan M, Livolsi VA. Encapsulated classic and follicular variants of papillary thyroid carcinoma: comparative clinicopathologic study. Endocr Pract. 2010;16(6):952-9.

24. Wang LY, Ghossein R, Palmer FL, Nixon IJ, Tuttle RM, Shaha AR, et al. Microscopic Positive Margins in Differentiated Thyroid Cancer Is Not an Independent Predictor of Local Failure. Thyroid. 2015;25(9):993-8.

25. Falvo L, Catania A, D'Andrea V, Marzullo A, Giustiniani MC, De Antoni E. Prognostic importance of histologic vascular invasion in papillary thyroid carcinoma. Ann Surg. 2005;241(4):640-6.

26. Gardner RE, Tuttle RM, Burman KD, Haddady S, Truman C, Sparling $\mathrm{YH}$, et al. Prognostic importance of vascular invasion in papillary thyroid carcinoma. Arch. Otolaryngol. Arch Otolaryngol Head Neck Surg. 2000;126(3):309-12.

27. Mete O, Asa SL. Pathological definition and clinical significance of vascular invasion in thyroid carcinomas of follicular epithelial derivation. Mod Pathol. 2011;24(12):1545-52.

28. Wreesmann VB, Nixon IJ, Rivera M, Katabi N, Palmer F, Ganly I, et al. Prognostic value of vascular invasion in well-differentiated papillary thyroid carcinoma. Thyroid. 2015;25(5):503-8.

29. Nikiforov YE, Ohori NP. Papillary carcinoma. In: Nikiforov YE, Biddinger PW, Thompson LDR (eds.). Diagnostic Pathology and Molecular Genetics of the Thyroid. 1st edition. Philadelphia, PA: Lippincott. 2012. p. 183-262.

30. Brennan MD, Bergstralh EJ, van Heerden JA, McConahey WM. Follicular thyroid cancer treated at the Mayo Clinic, 1946 through 1970: initial manifestations, pathologic findings, therapy, and outcome. Mayo Clin Proc. 1991;66(1):11-22.

31. Collini P, Sampietro G, Pilotti S. Extensive vascular invasion is a marker of risk of relapse in encapsulated non-Hürthle cell follicular carcinoma of the thyroid gland: a clinicopathological study of 18 consecutive cases from a single institution with a 11-year median follow-up. Histopathology. 2004;44(1):35-9.

32. Nishida T, Katayama Si, Tsujimoto M. The clinicopathological significance of histologic vascular invasion in differentiated thyroid carcinoma. Am J Surg. 2002;183(1):80-6.

33. Akslen LA, Myking AO, Salvesen H, Varhaug JE. Prognostic importance of various clinicopathological features in papillary thyroid carcinoma. Eur J Cancer. 1992;29A(1):44-51.

34. Simpson WJ, McKinney SE, Carruthers JS, Gospodarowicz MK, Sutcliffe SB, Panzarella T. Papillary and follicular thyroid cancer. Prognostic factors in 1,578 patients. Am J Med. 1987;83(3):479-88.

35. Mai KT, Khanna P, Yazdi HM, Perkins DG, Veinot JP, Thomas J, et al. Differentiated thyroid carcinomas with vascular invasion: a comparative study of follicular, Hürthle cell and papillary thyroid carcinoma. Pathology. 2002;34(3):239-44.

36. Hong JH, Yi HS, Yi S, Kim HW, Lee J, Kim KS. Implications of oncocytic change in papillary thyroid cancer. Clin Endocrinol (Oxf). 2016;85(5):797-804.

37. Pitoia, F., Ward, L., Wohllk, N., Friguglietti, C., Tomimori, E., Gauna, A., Camargo, R., Vaisman, M., Harach, R., Munizaga, F., Corigliano, S., Pretell, E., Niepomniszcze, H.: Recommendations of the Latin American Thyroid Society on diagnosis and management of differentiated thyroid cancer. Arq Bras Endocrinol Metabol. 2009;53:884-7. 